\title{
12 Anders denken und handeln - Bewusstsein für das Klima
}

\author{
Sabine Fritsch, Carolin Thiem, Oliver Sartori \\ Jede und jeder Einzelne kann einen Beitrag zu einer zukunftsfähigen und \\ nachhaltigen Entwicklung unseres Planeten leisten und den eigenen $\mathrm{CO}_{2}$-Fuß- \\ abdruck durch klimafreundliche Verhaltensweisen reduzieren. Warum nur fällt \\ das so schwer? Mangelt es am Bewusstsein?
}

In der Bevölkerungsumfrage „Umweltbewusstsein in Deutschland 2018" des Bundesministeriums für Umwelt, Naturschutz und nukleare Sicherheit (BMU) gaben 64 Prozent der Befragten an, dass das Thema Umwelt- und Klimaschutz eine "sehr wichtige Herausforderung" sei. Das waren elf Prozent mehr als in der gleichlautenden Umfrage des Jahres 2016 (Abb. 12.1, vgl. Rubik et al. 2019:9).

Um das Umweltbewusstsein in seiner Mehrdimensionalität zu erfassen, wurden die drei Teilbereiche Umweltaffekt, Umweltkognition und Umweltverhalten genauer betrachtet. Jedem dieser Teilbereiche lag ein Satz von Einstellungsaussagen oder Verhaltensselbstberichten zugrunde.

- Umweltaffekt: emotionale Reaktionen auf Umweltthemen werden betrachtet, zum Beispiel „Es macht mich wütend."

- Umweltkognition: Beurteilung sachlicher Aussagen zu Umweltthemen werden betrachtet, zum Beispiel zur Ressourcennutzung

- Umweltverhalten: Aussagen zum eigenen Verhalten in umweltrelevanten Bereichen des Alltags werden betrachtet, beispielsweise Einkauf, Mobilität

Die hieraus abgeleiteten Kennziffern zeigten auf einer Skala von 1 bis 10 für die Teilbereiche Umweltaffekt und Umweltkognition Werte von 7,2 bzw. 7,9. Das Umweltverhalten hingegen erreichte nur einen mittleren Wert von 4,6 und blieb damit hinter der affektiven und kognitiven Umwelteinstellung zurück (vgl. Rubik et al. 2019:67 ff.)

Die Umfrageergebnisse des BMU weisen darauf hin, dass das Klimabewusstsein in der Bevölkerung zunimmt. Ein Indikator dafür ist zum Beispiel die Bewegung „Fridays for Future", die seit 2018 weltweit Demonstrationen bzw. seit Ausbruch der CoronaPandemie Netzstreiks auslöst und sich damit für schnelle und effiziente Klimaschutzmaßnahmen einsetzt (vgl. Pohl 2020). 
Frage: Auf dieser Liste stehen verschiedene Probleme, denen sich unser Land heute gegenübersieht. Bitte geben Sie jeweils an, wie wichtig das Problem aus Ihrer Sicht ist. - Antwort: „sehr wichtig"

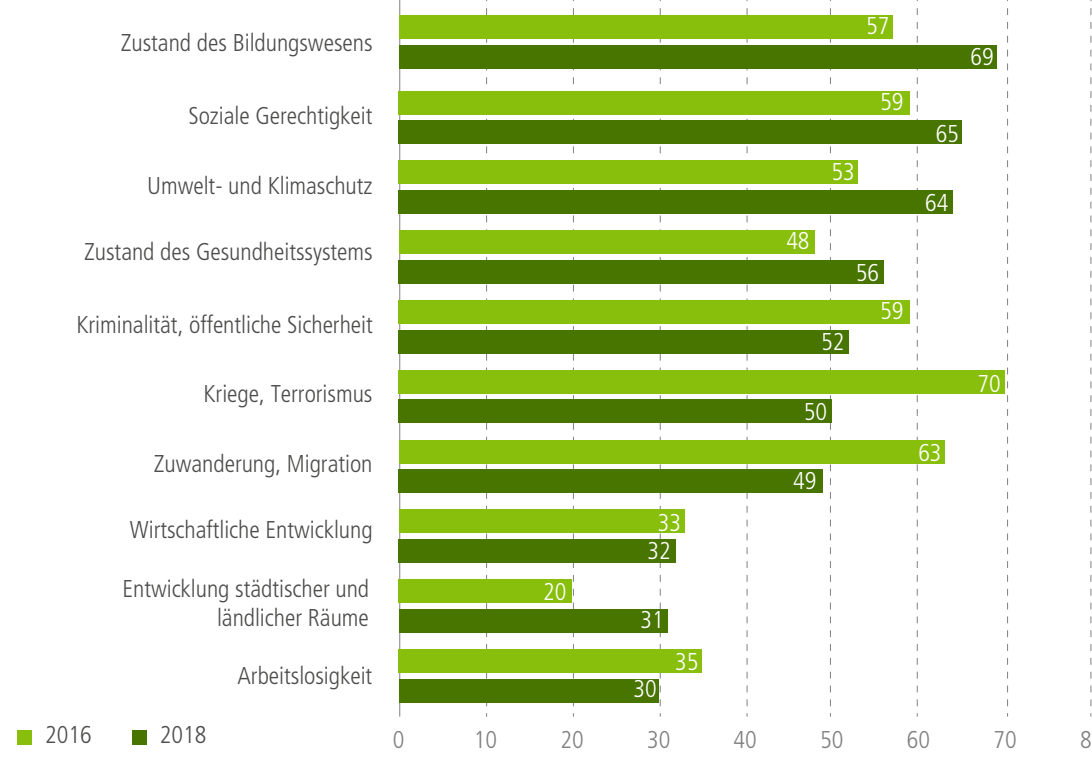

Repräsentativerhebung bei etwa 2.000 Befragten pro Erhebung (2018: Nacherhebung); Stichprobe ab 14 Jahren (Angaben in Prozent)

Abb. 12.1 Problemwahrnehmung der Bevölkerung in Deutschland 2016 und 2018. (Eigene Darstellung nach Rubik et al. 2019:17)

Gleichzeitig zeigt die Befragung des BMU aber auch, dass Klimabewusstsein nicht automatisch mit klimafreundlichem Verhalten gleichgesetzt werden kann. In der Psychologie ist das Phänomen schon lange Zeit als "Attitude-Behaviour-Gap" bekannt. Es beschreibt die Diskrepanz zwischen dem geplanten und dem tatsächlichen Verhalten. Die Ursachen dafür liegen in einer Vielzahl von Faktoren begründet, die zum Beispiel durch persönliche Wertvorstellungen, sozialen und gesellschaftlichen Druck, das Gefühl von Kontrolle und Erfahrungswerte beeinflusst werden. So werden beispielsweise Konsumentscheidungen nicht zweckrational getroffen, sondern unterliegen sozialen, ökonomischen und psychologischen Einflüssen. Der Mensch befindet sich dabei kontinuierlich in einem Konflikt zwischen Eigeninteresse und Gemeinschaftsinteresse. Je größer dieses Konfliktpotenzial ist - je größer die Differenz zwischen dem kurzfristigen, persönlichen Interesse und dem langfristig orientierten Gemeinwohl (beispielsweise Klima- und Umweltschutz) -, desto geringer ist der tatsächliche Einfluss des vorhandenen Umweltbewusstseins auf die aktiven Handlungen (vgl. Haubach et al. 2013:46 f.). 
Dabei stellt die Risikowahrnehmung des Menschen für das Eigeninteresse eine wichtige Einflussgröße dar. Werden das Risiko und die Bedrohung durch den Klimawandel als hoch wahrgenommen, steigt das Bewusstsein für die Thematik. Menschen, die häufig und wiederkehrend persönlich durch Wetterkatastrophen wie zum Beispiel Überschwemmungen oder extreme Hitzewellen betroffen sind, neigen zu einem höheren Bewusstsein. Eigeninteresse und Gemeinschaftsinteresse nähern sich an mit dem Resultat eines eher klimafreundlichen Verhaltens. Im Gegensatz dazu resultiert aus einer mittelbaren Betroffenheit - in der Klimafrage trifft dies auf einen Großteil der Weltbevölkerung zu - die Abwertung des Risikopotenzials und das Empfinden einer geringen Bedrohung (vgl. Haubach et al. 2013:44 f.).

Die Abwertung von Risikopotenzialen und die damit verbundene Abnahme von klimafreundlichem Verhalten lassen sich maßgeblich durch die Wirksamkeit sozialökonomischer Faktoren erklären. Dazu zählen beispielsweise die Diskontierung künftiger Schäden oder das Gefangenendilemma aus der Spieltheorie. Die Diskontierung beschreibt, wie zukünftige Gefahren zu einem gegenwärtigen Zeitpunkt als wesentlich geringer eingeschätzt werden. Der Klimawandel zeichnet sich jedoch nicht nur auf einem langfristigen Zeithorizont ab, sondern ist in Deutschland mit heißen Sommern und Gletscherschmelze in den Alpen bereits deutlich sichtbar. Allerdings verteilen sich seine Wirkungen und die Häufigkeit von extremen Ereignissen weltweit und treten unregelmäßig in Erscheinung. Für den einzelnen Menschen ist er deshalb rational schwer greifbar und wenig nachvollziehbar. Tatsächlich jedoch ist der Klimawandel manifest - mit schleichenden Auswirkungen auf den Alltag und teilweise schon mit dramatischen Konsequenzen. Zugleich sind die Kosten, die durch das Unterlassen von Maßnahmen zum Klimaschutz entstehen und daraus resultierende Umweltschäden, kaum zu ermitteln und somit in unserem monetären Wertesystem nicht darstellbar (vgl. Rogall 2012:79 f.).

Das Gefangenendilemma der Spieltheorie kennzeichnet eine Situation, in der individuell rationales Verhalten jedes einzelnen Gruppenmitgliedes zu einem für die Gruppe insgesamt nicht optimalen Ergebnis führt. Im Kontext des Klimawandels fällt es Individuen schwer, Verhaltensänderungen umzusetzen, die zwar im Ergebnis dem Gemeinwohl förderlich sind, allerdings der einzelnen Person als Verzicht empfunden werden und somit negativ konnotiert sind. Vor allem dann, wenn die einzelne Person nicht sicher ist, dass alle anderen ebenfalls ihr Verhalten ändern, schlägt die Falle des Gefangenendilemmas zu (vgl. Rogall 2012:78). Wird der eigene Beitrag außerdem als gering oder effektlos wahrgenommen oder werden individuelle Handlungsmöglichkeiten nicht erkannt, wird klimafreundliches Verhalten immer unwahrscheinlicher. 


\section{Bewusstsein schaffen durch Partizipation}

Gegenwärtig ist es vor allem die von Schülerinnen und Schülern getragene Bewegung "Fridays for Future", die regelmäßig und unablässig auf die bedrohliche Umweltsituation auf unserer Erde aufmerksam macht. Für Deutschland fordert „Fridays for Future" unter anderem, unverzüglich alle Subventionen für fossile Energieträger wie Kohle, Öl und Gas zu streichen sowie ein Viertel der Kohlekraft abzuschalten. Der Bewegung haben sich nach und nach weitere Gruppen angeschlossen. So gibt es mittlerweile etwa die Bewegungen "Parents for Future" und "Scientists for Future". Die Geschichte lehrt, dass Veränderungen oft mithilfe von sozialen Bewegungen also einer Form uneingeladener Beteiligung (vgl. Wehling 2012) - und somit bewusstem Handeln erkämpft werden konnten, meist gegen heftigen Widerstand in der etablierten Politik.

Kritik an bestehenden Strukturen wird nicht immer gleich begrüßt, aber im Hinblick auf Partizipation fördert die Politik sie nicht nur, sondern fordert sie auch ein. Demokratie soll aktiv gelebt werden. Dies findet sich etwa in politischen Roadmaps wieder, in denen Partizipation nicht nur ein Kapitel füllt, sondern zentraler Bestandteil ist (vgl. Bundesministerium für Bildung und Forschung 2015; Bundesministerium für Bildung und Forschung 2016). In den letzten Jahren wurden Formen der Partizipation am demokratischen Geschehen stetig weiterentwickelt, sodass es neben dem Engagement in einer Partei inzwischen zahlreiche weitere Möglichkeiten gibt, sich in den unterschiedlichsten Initiativen politisch zu engagieren - ob für ein klimarelevantes Ziel wie die Aufforstung, für die Vermeidung von Plastikmüll oder für fahrradfreundliche Innenstädte.

In den vergangenen Jahren haben sich einhergehend mit der Entwicklung digitaler Technologien weitere Partizipationsmodi herausgebildet. Unterschieden wird hierbei unter anderem zwischen informellen und formellen E-Partizipationsverfahren. Formelle Verfahren des „E-Aktivismus" fördern schwerpunktmäßig Diskussion und Austausch und sollen den Prozess der Meinungsbildung unterstützen. Sie fungieren auch als Gegengewicht zu informellen Diskussionen in den sozialen Medien. Diese Verfahren können in Fragen des Klimaschutzes ggf. helfen, auch Akzeptanz für politische Entscheidungen zu schaffen, die unser Leben zwar einschränken, aber der weiteren Erderwärmung entgegenwirken.

Informelle Verfahren zielen meist auf Entscheidungsfindungen ab (zum Beispiel Online-Petitionen oder internetbasierte Abstimmungen über Bauvorhaben oder ähnliche). Im Bereich Klima nutzt beispielsweise Greenpeace die Plattform "greenaction". Hier kann über Texte, Videos und Bilder auf Themen aufmerksam gemacht, zu Demonstrationen aufgerufen oder Geld gesammelt werden. Registrierte Nutzende haben die Möglichkeit, mit wenigen Klicks Petitionen zu unterzeichnen, zu spenden oder ihr Engagement öffentlich sichtbar zu machen. Es wird außerdem er- 
möglicht, vorbereitete E-Mails an Freunde zu versenden oder Logos und Bilder von Kampagnen auf Social-Media-Profilen zu veröffentlichen. Es handelt sich sozusagen um eine digitale Version von Flugblatt und Anstecknadel.

Diese Art der digitalen Petitionen verschafft Individuen die Möglichkeit, Unterstützung für ein Anliegen oder Vorhaben innerhalb kürzester Zeit und mit wenig Aufwand kundzutun. In diesem Zusammenhang etablieren sich die Begriffe Slacktivismus (vgl. Morozov 2012) und Clicktivismus (vgl. Halupka 2014). Slacktivismus ist eine Zusammensetzung aus dem englischen Wort "Slacker" - eine Arbeit oder Anstrengung vermeidende Person - und "Aktivismus" (Morozov 2012). Was heißt das genau? Durch Begriffe wie Slack- und Clicktivismus wird der Umstand angeprangert, dass die Inhalte von politisch motivierten Initiativen oder Personen im Digitalen zwar häufig gelikt, geteilt oder kommentiert und entsprechend weiterverbreitet werden. Jedoch wirkt sich diese Form des Engagements wenig auf politisches Geschehen aus und oft bleibt weiteres politisches Engagement aus - so kritische Stimmen (vgl. ebd.).

Durch das farbliche Ändern eines Profilbildes oder das Liken einer Aktivität, wie beispielsweise einer "Fridays-for-Future"-Demonstration, können Solidarität und politische Meinungen öffentlich gemacht werden. Allerdings verlangt das Liken oder das Verändern eines Profilbildes laut des Autoren (vgl. ebd.) vom Individuum eigentlich keine weitere Auseinandersetzung mit dem jeweiligen politischen Thema. Ohne Aufwand oder Mühe kann es sich als "Gutmensch" in seinen sozialen Netzwerken darstellen. Deshalb wird diese Art der Beteiligung von vielen stark kritisiert und es gibt es auch Stimmen, die dadurch politische Beteiligung generell infrage gestellt sehen (vgl. Cuéllar 2017).

Slack- und Clicktivismus sind Ausdruck der eingangs erwähnten Diskrepanz zwischen geplantem und tatsächlichem Verhalten für das Gemeinwohl. Denn ein Klick bedeutet noch längst nicht, dass das eigene Handeln bewusst klimaschonender wird. Es kann aber dazu führen, dass zumindest ein Bewusstsein dafür geschaffen wird, dass Veränderungen nötig sind. Positiv betrachtet könnte formuliert werden: Wenig Auseinandersetzung ist auch eine Art der Auseinandersetzung. Wissenschaftliche Untersuchungen zu diesen Partizipationsmodi stehen noch ganz am Anfang. Es deutet sich jedoch an, dass Fragen zur Rolle von digitaler Beteiligung im Rahmen der Klimadebatte es wert zu sein scheinen, mehr Aufmerksamkeit zu erhalten.

Neben neuen digitalen Beteiligungsformaten sind in den vergangenen Jahren auch andere innovative Formate wie die sogenannten "Hackathons" entstanden, die sich neuen Technologien widmen. Das aus "Hacking" und "Marathon" bestehende Kunstwort Hackathon beschreibt eine zeitlich begrenzte Veranstaltung zur kollaborativen Konzeption von Lösungsansätzen für eine klar definierte Herausforderung. Geprägt wurde der Begriff in der Hard- und Softwareentwicklung Ende der 1990erJahre, aber spätestens seit Mitte der 2000er-Jahre erfolgte mit dem Civic Hacking 
die Anwendung auch auf gesellschaftliche Fragestellungen wie den Klimawandel. Im Rahmen des Projekts "Green Hack" entwickelten Jugendliche beispielsweise eine App, die zu eigenen Klimaschutzaktivitäten motivieren soll. Durch die Auseinandersetzung mit dem Thema während der App-Entwicklung konnte auf ganz neue Art ein Bewusstsein für das Thema Klima geschaffen werden. Im verlängerten Wissenschaftsjahr zu Ozeanen und Meeren 2016/2017 wurden Ozeanwerkstätten durchgeführt, die ebenso als Civic Hackathons begriffen werden können. Im Jahr 2019 veranstaltete die Universität Bochum den Climathon Ruhr, bei dem Lösungen zum Umgang mit dem Klimawandel entwickelt werden sollten. All dies sind Beispiele einer inzwischen endlos langen Liste.

Durch die Wissensaneignung während eines Hackathons kann Bewusstsein dafür entstehen, dass die vom Individuum im Alltag erlebten Zustände in Zusammenhang mit dem Klimawandel stehen - und dass mit klimafreundlichem Handeln verbundene Einschränkungen in den nächsten Jahren nötig werden. Ebenso kann mit diesem Format der Beteiligung das Individuum dem Ohnmachtsgefühl gegenüber der übergroßen Herausforderung Klimawandel entgegentreten (vgl. Thiem 2020 [im Druck]). Da es sich bei diesem Format um ein sehr junges Phänomen handelt, steckt die Forschung dazu ebenfalls in den Anfängen. Eine wichtige Frage ist, ob die Ergebnisse von Hackathons tatsächlich weiterverfolgt werden und welche langfristigen Handlungseffekte sich bei den Beteiligten von Klima-Hackathons einstellen.

Mehr Nachhaltigkeitsbewusstsein im Kontext von Handlungsveränderungen wird aktuell eher von langfristigen Beteiligungsformaten erwartet. So veranstaltete die Region Tirol 2016 einen Ideenwettbewerb und begleitete die Teilnehmenden über zwei Monate hinweg mit einem Mentoring, mit Workshops und einer CrowdfundingKampagne auf ihrem Weg, ihre Ideen in die Tat umzusetzen.

Über den Ideenkanal, so verrät es die Website, ist auch eine soziale Innovation, die ihren Ursprung in Deutschland hat, nach Liechtenstein diffundiert. Denn eine Ideengeberin möchte dort die sogenannte "GemüseAckerdemie" etablieren. Dabei handelt es sich um ein Bildungsprogramm, das Kindern und Jugendlichen die Themen Lebensmittelproduktion und -wertschätzung, gesunde Ernährung und Nachhaltigkeit näherbringt. Das Konzept ist skalierbar und wurde bereits vielfach (665-mal in Kitas und Schulen, Stand: Mai 2020) im deutschsprachigen Raum umgesetzt; die GemüseAckerdemie gilt als soziale Innovation. Darüber hinaus sind in den vergangenen Jahren vielfältige neue Bildungsangebote entstanden, die auf den Konsum von regionalen nachhaltigen Produkten abzielen („Marktschwärmer", , etepetete Retterboxen", "Solidarische Landwirtschaft"). Es sind diese Ideen, die bereits im Kindesalter aber, aber auch darüber hinaus, den Grundstein dafür legen können, verändertes Handeln im Sinne des Klimaschutzes nicht als lästige Notwendigkeit wahrzunehmen, 
sondern für einen tatsächlichen Wertewandel zu sorgen. In Weiterverfolgung dieses Ansatzes kann eine neue, klimabewusste Generation heranwachsen.

\section{Klimabewusste soziale Innovationen und Social Enterprises}

Soziale Innovationen umfassen neue soziale Praktiken und Organisationsmodelle, die darauf abzielen, für die Herausforderungen unserer Gesellschaft tragfähige und nachhaltige Lösungen zu finden (vgl. BMBF 2018:11). Auch die Politik entdeckt zunehmend dieses Handlungsfeld. Dementsprechend hat das "Hightech Forum" (2019) - das zentrale Beratungsgremium der Bundesregierung - sich der Förderung von sozialen Innovationen angenommen und ein Impulspapier zu diesem Thema verfasst.

Der europäische Green Deal (2019) nimmt zwar nicht explizit Bezug auf soziale Innovationen, obwohl diese gerade in den Bereichen Mobilität, Energie und nachhaltiger Konsum vorzufinden sind. Allerdings veranstaltete die EU 2020 die Social Innovation Competition zum Thema Sustainable Fashion. Auf der Website (European Social Innovation Competition, 2020) heißt es: „Der Verbrauch von Textilien macht etwa 5 Prozent des ökologischen Fußabdrucks der EU aus, und Bekleidung und Schuhe sind für etwa 8 Prozent der globalen Treibhausgasemissionen weltweit verantwortlich. Pro Jahr kaufen die Bürger:innen der EU jeweils mehr als 12 Kilogramm Kleidung, deren Herstellung 195 Millionen Tonnen $\mathrm{CO}_{2}$ in die Atmosphäre abgegeben und 46 Milliarden Kubikmeter Wasser verbraucht hat. Es ist Zeit, etwas zu ändern!" Der Wille, mithilfe sozialer Innovationen Veränderungen hervorzurufen, ist demnach in der EU vorhanden.

Soziale Innovationen können sich in neuen Produkten oder Dienstleistungen sowie in neuen Arbeits- und Produktionsprozessen oder Kooperationsformen zeigen. Für jede dieser Kategorien lassen sich bereits Beispiele in der Praxis finden, die für den Klimawandel relevant sind. Innovative Kooperationsformen sind beispielsweise Initiativen, die Urban Gardening betreiben und so zum einen die Luftqualität in den Städten verbessern und zum anderen regionale Lebensmittelproduktion fördern. Bioenergiedörfer und andere Formen der Energiegenossenschaften zeigen beispielsweise, wie nachhaltige Energieproduktion auch einen im Kleinen spürbaren wirtschaftlichen Mehrwert bringen kann (vgl. Strigl 2014).

Soziale Innovationen schließen außerdem wirtschaftlichen Erfolg nicht aus (vgl. G.I.B. - Gesellschaft für innovative Beschäftigungsförderung mbh NRW 2010). Das zeigt zum Beispiel das Unternehmen „Original Unverpackt". Früher ist es belächelt worden, aber mittlerweile gibt es mehr als 100 Unverpackt-Supermärkte in ganz Deutschland, in denen bewusst nachhaltig konsumiert wird. Soziale und technische Innovationen gehen zudem oft Hand in Hand wie beispielsweise "SpraySafe", ein 
genusstaugliches Schutzspray zur Anwendung auf Lebensmitteln, das den Bedarf an Plastikverpackungen und -behältnissen reduziert. Schon diese wenigen Beispiele illustrieren eindrücklich: Es ist wichtig, stets neben technischen Innovationen auch soziale Innovationen als Lösungsmöglichkeit für Klimafragen mitzudenken und in Erwägung zu ziehen.

In diesem Zusammenhang sind auch die Leistungen von Social Entrepreneuren im Kampf gegen die Folgen der globalen Erderwärmung von Bedeutung. Unternehmen wie "Ecosia", eine nachhaltige Suchmaschine, oder "Polarstern", ein nachhaltiger Stromanbieter, zeigen zum Beispiel, wie sich Unternehmen gemeinwohlorientiert für den Klimaschutz einsetzen können und Alternativen anbieten. Dass für Sozialunternehmen der Zugang zu Finanzierungs- und Förderinstrumenten der öffentlichen Hand immer noch erschwert ist, ist Gegenstand politischer Auseinandersetzung. Diskutiert wird außerdem, ob etwa nachrichtenlose Konten im Rahmen eines Impact Bonds als Finanzierungsinstrument für Social Entrepreneure genutzt werden könnten (vgl. Sauerhammer et al. 2019).

Die grundlegende Frage ist, wie die beschleunigte globale Erderwärmung gestoppt und umgekehrt werden kann und an welcher Stelle engagierte Bürger:innen Lösungen erarbeiten und umsetzen könnten. Die entsprechenden Fragen für die Wirtschaft - und somit auch für Sozialunternehmen - lauten: Welche Bedürfnisse ergeben sich aus den nötigen Anpassungen, für die ich Lösungen anbieten kann, und wie muss ich mein Modell für eine bessere Klimabilanz aktualisieren? Für die Politik stellt sich die Frage, welche Art der Unterstützung seitens der Demokratie für Bürgerinnen und Bürger aber auch für Angebote aus der Wirtschaft nicht nur ermöglicht, sondern auch eingefordert werden muss.

Zu den Herausforderungen, die soziale Innovationen mit sich bringen, gehört vor allem die Identifikation von Maßstäben für den Erfolg. Gängige Indikatoren für technischen Erfolg sind nämlich nicht ohne Weiteres auf soziale Innovationen übertragbar. In Bezug auf den Klimawandel stellt sich somit die Frage, ob und wie $\mathrm{CO}_{2}$-Einsparungen bei der Verbreitung von sozialen Innovationen gemessen und dingfest gemacht werden können. Ein Umdenken innerhalb der Wirtschaft ist sicherlich vonnöten, denn oftmals werden ausschließlich finanzielle Kennzahlen zu Rate gezogen, nicht aber die Frage aufgeworfen, ob ein Unternehmen ökologisch oder sozial handelt. Dieses Umdenken lässt sich unter dem Label Neoökologie zusammenfassen. Auf diese Weise entwächst Nachhaltigkeit individuellem Lifestyle, wird zur gesellschaftlichen Bewegung und kann den Konsumtrend zum Wirtschaftsfaktor machen (vgl. Anthes 2020). 


\section{Umweltbewusstsein durch interaktive Technologien}

Neben sozialen Innovationen können auch intelligente interaktive Technologien helfen, das Umweltbewusstsein von Menschen zu stärken, Klimazusammenhänge zu erklären und zu Verhaltensänderungen zu motivieren. Es gibt schon Apps fürs Smartphone, die potenzielle Folgen des Klimawandels anschaulich und greifbar machen. "Studioresilience" nutzt zum Beispiel Augmented Reality, um das Bild von Straßenszenen entsprechend des prognostizierten anwachsenden Meeresspiegels mit Wasser zu überlagern oder um in Küstenbilder abgestorbene Korallenriffe einzublenden (vgl. Sacher 2020). Tatsächlich unterstützt schon eine Vielzahl von Apps und Onlinetools die Bürgerin oder den Konsumenten beim klimagerechten Verhalten. Angezeigt werden etwa Nahrungsmittel von Geschäften "in deiner Umgebung", die verteilt werden, um nicht weggeworfen zu werden. Inhaltsstoffe von Nahrungsmitteln und Kosmetika werden entschlüsselt. Beim Konsum wird man durch ökologische Ratschläge unterstützt und für die nachhaltige Mobilität kann man verschiedenste Leihfahrzeuge buchen oder eine Mitfahrgelegenheit finden. Der Energieverbrauch zu Hause kann per Smartphone gehandhabt und die individuelle $\mathrm{CO}_{2}$-Bilanz erfasst werden (vgl. Otto [GmbH \& Co KG] 2020).

Insgesamt steht eine Vielzahl an Einzellösungen für die Lebensbereiche Wohnen, Konsum, Ernährung und Mobilität zur Verfügung. $\mathrm{CO}_{2}$-Rechner, die auf vielen Internetseiten angeboten werden, schlüsseln den eigenen $\mathrm{CO}_{2}$-Beitrag nach diesen Lebensbereichen auf. Dazu werden die persönlichen Parameter mehr oder weniger detailliert abgefragt („Wie groß ist Ihr jährlicher Stromverbrauch?", "Wie oft essen Sie Fleisch?" etc.). Der so verschaffte Überblick bietet den Nutzer:innen die Möglichkeit, Sünden oder Heldentaten beim Fleischkonsum oder Pkw-Verzicht zu vergleichen und zu gewichten. In solchen Angeboten wird der eigene $\mathrm{CO}_{2}$-Fußabdruck mit dem nationalen oder globalen Durchschnitt oder mit dem idealen, also echt nachhaltigen, individuellen $\mathrm{CO}_{2}$-Fußabdruck verglichen (s. Abb. 12.2).

Der $\mathrm{CO}_{2}$-Rechner des Umweltbundesamtes beispielsweise kategorisiert nach Heizung und Strom, Mobilität, Ernährung, Konsum und öffentlichen Emissionen. Bei der Erhebung kann die Detailtiefe angepasst werden. Das Ergebnis wird im Verhältnis zum deutschen Durchschnitt dargestellt. Optimierungsszenarien zeigen den Weg zu einer $\mathrm{CO}_{2}$-ärmeren individuellen Zukunft. Hiermit steht ein relativ funktionales Werkzeug zur Verfügung, mit dem man mit einigermaßen überschaubarem Aufwand sein Wissen erweitern kann und einen groben Überblick über den eigenen Beitrag zum globalen $\mathrm{CO}_{2}$-Ausstoß erhält (vgl. KlimAktiv 2018).

Noch sind der $\mathrm{CO}_{2}$-Rechner und die Vielzahl vorhandener Apps statische Werkzeuge, die eine manuelle Eingabe erfordern, sich nicht aktualisieren und Veränderungen im Verhalten nicht verfolgen können. Sie helfen letztlich nicht wirklich dabei, die Diskrepanz zwischen Umweltbewusstsein und Umweltschutzverhalten aufzulösen. 


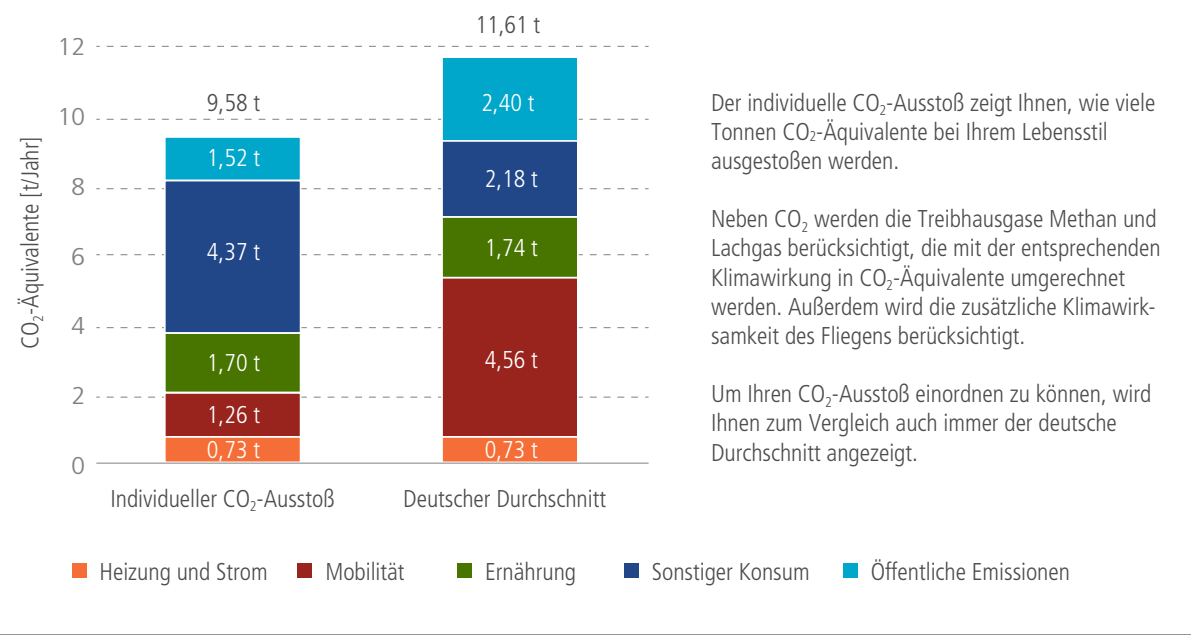

Abb. 12.2 Individueller $\mathrm{CO}_{2}$-Ausstoß als Ergebnis des Online-CO2-Rechners des Umweltbundesamts (https://uba.co2-rechner.de/de_DE/?bookmark=EtfVR2FdQmLNHumO, zuletzt geprüft am 10.07.2020.). (Eigene Darstellung)

Sie zeigen zwar das Problem auf, bieten aber in dem Moment des größten Problembewusstseins keine praktische Lösung an.

Die Hürden für ein klimagerechtes Verhalten sind weiterhin hoch. Der eigene Beitrag scheint in Relation zur Größe des Problems verschwindend klein, die Klimaveränderungen sind schleichend und die Zusammenhänge zwischen Klimawandel und dem eigenen Heiz-, Mobilitäts- oder Konsumverhalten sind komplex und vielschichtig. Uns fehlen sowohl Informationen als auch Motivation. Wir haben noch kein Konzept im Sinne von Wert und Verfügbarkeit für die Ressource $\mathrm{CO}_{2}$ entwickeln und verinnerlichen können, wie wir das etwa für die Ressourcen Geld oder Zeit getan haben. Daher fällt uns der Umgang mit $\mathrm{CO}_{2}$ schwer (vgl. Franke 2019). Hier können interaktive Technologien von Nutzen sein. Intelligente Sensorik, mobile Systeme und Werkzeuge der Datenanalyse können aus statischen Systemen intelligente individuelle Assistenzsysteme machen, die uns die Problemzusammenhänge angemessen erläutern und uns bei der Lösung zielführend und motivierend unterstützen.

Mittel- und langfristig werden sicherlich funktionale Assistenzsysteme entstehen, die hier Hilfe und Unterstützung bieten. Durch deren Einsatz werden die Nutzenden klimarelevante Zusammenhänge verstehen können; sie gewinnen so an Klimasouveränität. Solche Assistenzsysteme sollten erstens den individuellen $\mathrm{CO}_{2}$-Beitrag durch sensorische Erfassung und/oder Zugriff auf individuelle Daten in angemessener Genauigkeit erfassen und darstellen. Hierbei sind Fragen des Datenschutzes zu beantworten, um Vertrauen und Akzeptanz zu schaffen. Auch sollte der individuelle 
$\mathrm{CO}_{2}$-Beitrag in angemessener Weise in einen globalen Zusammenhang gestellt werden. Die vergleichende Darstellung mit der Nachbarschaft, Menschen in anderen Regionen, dem Weltdurchschnitt und dem Wert, der für Nachhaltigkeit erforderlich ist, ermöglicht die individuelle Einordnung und Bewertung. Dabei sollte nicht nur der aktuelle, sondern auch der historische $\mathrm{CO}_{2}$-Beitrag berücksichtigt werden, der Europa und Nordamerika noch deutlich schlechter dastehen lässt. Die globale Klimagerechtigkeit sollte thematisiert werden, verschiedene Klimaszenarien sollten entworfen und dafür erforderliche Budgets abgeleitet werden. Das Erreichen eines individuellen $\mathrm{CO}_{2}$-Zielwertes kann sehr motivierend sein.

Psychologische Versuche zeigen, dass Handlungswissen am ehesten praktisch genutzt wird, wenn es auf die individuellen Bedingungen zugeschnitten ist (vgl. Hamann 2016). Daher sollten die Assistenzsysteme zweitens individuelle Handlungsvorschläge mit Einsparszenarien machen und die Nutzerinnen und Nutzer bei der Gestaltung eines eigenen $\mathrm{CO}_{2}$-Fahrplans unterstützen. Die eine verzichtet leichter auf Fleisch, der andere kann sich den Wocheneinkauf ohne Auto schwer vorstellen, wieder anderen fällt es leichter, sich auf Ökostromanbieter einzulassen.

Hier sind konkrete und realisierbare Vorschläge wichtig, die sowohl technische als auch soziale Innovationen beinhalten. Hürden für die Umsetzung sollen diese Systeme identifizieren können und möglichst direkt beseitigen. Als ein gelungenes Beispiel kann eine Leckage-App gelten: Diese Anwendung ermittelt die monetären Kosten von Druckluftverlusten und stellt innen die Kosten für die Reparatur der Leckage gegenüber. Meist legt dieser Vergleich eine unverzügliche Reparatur nahe. Den Moment der Erkenntnis nutzend benennt die App die notwendigen Ersatzteile und bietet an, diese sofort zu bestellen (vgl. LOOXR GmbH 2019).

Drittens sollen geeignete Verfahren für das Tracking des eigenen Umweltverhaltens entwickelt, angeboten und ggf. auch steuerlich unterstützt werden. So können auch Menschen ohne ausgeprägtes Klimabewusstsein erreicht werden. Neben dem Monitoring des Mobilitätsverhaltens über die Position des Smartphones (GPS, Beschleunigung etc.) könnten die Daten des Zahlungsverkehrs für das Konsumverhalten, Verbrauchsdaten (Smartmeter u. a.) für den Ressourcenverbrauch zu Hause, intelligente Kühlschränke oder Biosensoren für das Ernährungsverhalten von neuen, funktionalen Assistenzsystemen ausgewertet werden. Ziel sollte eine übersichtliche Darstellung der Einsparungen sein. Zudem sollten die Ergebnisse so präsentiert sein, dass sie individuelle "Motivationsmechanismen" unterstützen. Wichtig ist hier die Berücksichtigung aller Lebensbereiche. Die im Systemdesign verankerte Sicherheit der Daten ist Grundvoraussetzung, um Akzeptanz und Vertrauen zu schaffen.

So entstehen echte intelligente Umweltassistenten, die Verhaltensoptionen und Entscheidungshilfen auf allgemeiner Ebene oder in sehr konkreten Situationen (Burger vs. Sushi, Plastik- vs. Papiertüte, Wärmepumpe vs. Gasbrennwertkessel etc.) an- 
bieten. Dabei können sie Situation und Kontext berücksichtigen (bei Regen ist die Plastiktüte sinnvoller, für den Wocheneinkauf ist das Taxi besser als das Leihrad). Sie vermitteln und erklären die komplexen Verhalten-Klima-Zusammenhänge und bieten im Ergebnis eine realistische Chance auf nachhaltige $\mathrm{CO}_{2}$-Reduktion.

Die Handlungsfelder Partizipation, soziale Innovation und interaktive Technologien können nicht isoliert voneinander bearbeitet und vorangebracht werden, denn sie funktionieren nur im Zusammenspiel. Mit Partizipationsmethoden können soziale Innovationen entstehen, die auf neuer Technologie beruhen. Gleichzeitig können soziale Innovationen partizipative Prozesse und die Weiterentwicklung von Technologien anstoßen. Technische Systeme wiederum müssen nutzerzentriert entwickelt werden und soziale Innovationen berücksichtigen und einbeziehen. Nur in Kombination miteinander führen die Handlungsansätze zu einem breiteren und tieferen Umweltbewusstsein, das so beschaffen ist, dass notwendige klimarelevante Anpassungen leichter akzeptiert werden können.

Neben individuellen $\mathrm{CO}_{2}$-Einsparungen wird der größere Klimaeffekt durch informierte, klimabewusste und aktive Bürger:innen erreicht. Sie können das Klima- und Umweltbewusstsein gesellschaftlich und politisch multiplizieren. Vor allem im Zusammenspiel der skizzierten Maßnahmen lassen sich Synergieeffekte vermuten. Gleichwohl ist zu erwarten, dass Menschen, die bisher keine Relevanz in der Debatte gesehen haben, auch mit diesen Maßnahmen nur schwer erreicht werden können. Letztlich sind deshalb auch regulative Maßnahmen (zum Beispiel Flugverbote) und die Anpassung wirtschaftlicher Angebote (klimaneutrale Produkte und Services) notwendig. 


\section{Literatur}

Anthes, Dan (2020): Wie Nachhaltigkeit in den 2020ern zum Businessstandard wird. Verfügbar unter https://www.xing.com/news/klartext/wie-nachhaltigkeit-in-den-2020ern-zumbusinessstandard-wird-3603, zuletzt geprüft am 17.04.2020.

Bundesministerium für Bildung und Forschung (Hrsg., 2015): Zukunftsstadt - Strategische Forschungs- und Innovationsagenda. Verfügbar unter https://www.fona.de/medien/pdf/ Zukunftsstadt.pdf, zuletzt geprüft am 21.09.2020.

Bundesministerium für Bildung und Forschung (Hrsg., 2016): Grundsatzpapier Partizipation.

Bundesministerium für Bildung und Forschung (Hrsg., 2018): Forschung und Innovation für die Menschen. Die Hightech Strategie 2025. Verfügbar unter https://www.hightech-strategie.de/files/HTS2025.pdf, zuletzt geprüft am 02.05.2020.

Cuéllar, Lya (2017): Klicktivismus: Reichweitenstark aber unreflektiert?, Bundeszentrale für politische Bildung. Verfügbar unter https://www.bpb.de/lernen/digitale-bildung/ werkstatt/258645/klicktivismus-reichweitenstark-aber-unreflektiert, zuletzt geprüft am 13.08.2020.

Dr. Rubik, Frieder; Müller, Ria; Harnisch, Richard; Dr. Holzhauer, Brigitte; Schipperges, Michael; Dr. Geiger, Sonja (2019): Umweltbewusstsein in Deutschland 2018. Bundesministerium für Umwelt, Naturschutz und nukleare Sicherheit; Umweltbundesamt (Hrsg.).

European Social Innovation Competition (2020): Reimagine Fashion. Verfügbar unter https:// eusic.challenges.org/2019-theme/, zuletzt geprüft am 02.05.2020.

European Commission (2019): Communication from the Commission. The European Green Deal. Verfügbar unter https://eur-lex.europa.eu/legal-content/EN/TXT/?qid=1588580774040\&uri=CELEX, zuletzt geprüft am 13.08.2020.

Franke, Thomas (2019): „YouTube: Future Energies Science Match 2019", Wie wir den Klima-Analphabetismus überwinden. Verfügbar unter www.youtube.com/watch?v=P_ ITFdlyrvg\&list=PLOEQYY4L5K7toP4ezOECW4e6-ONoxGrzz\&index=56, zuletzt geprüft am 07.05.2020.

G.I.B. - Gesellschaft für innovative Beschäftigungsförderung mbh NRW (2010): Soziale Innovationen haben eine hohe wirtschaftliche Relevanz - Interview mit Jürgen Howaldt. Verfügbar unter https://www.gib.nrw.de/service/downloaddatenbank/interview-mit-profdr-juergen-howaldt, zuletzt geprüft am 02.05.2020.

Halupka, Max (2014): Clicktivism: A Systematic Heuristic. Policy \& Internet, 6(2), 115-132. Verfügbar unter https://doi.org/10.1002/1944-2866.POI355, zuletzt geprüft am 13.08.2020.

Hamann, Karen; Baumann, Anna; Löschinger, Daniel (2016): Psychologie im Umweltschutz Handbuch zur Förderung nachhaltigen Handelns. München: oekom.

Haubach, Christian; Moser, Andrea; Schmidt, Mario; Wehner, Christa (2013): Die Lücke schließen - Konsumenten zwischen ökologischer Einstellung und nicht-ökologischem Verhalten. In: Wirtschaftspsychologie, Heft 2/3-2013, 43-57. 
Hightech Forum (2019): Soziale Innovationen. Ein Impulspapier für das Hightech Forum. Verfügbar unter https://www.hightech-forum.de/wp-content/uploads/hightech-forum_impulspapier_soziale_innovationen.pdf, zuletzt geprüft am 02.05.2020.

KlimAktiv. (2018): $\mathrm{CO}_{2}$-Rechner, verfügbar unter https://uba.co2-rechner.de, zuletzt geprüft am 07.05.2020

LOOXR GmbH. (2019): Smartes Leckagemanagement, verfügbar unter www.looxr.de/leckagemanagement/, zuletzt geprüft am 07.05.2020.

Morozov, Eygeny (2012). New delusion. The dark side of internet freedom. New York: Public Affairs.

Otto (GmbH \& Co KG) (2020): Umwelt-Apps: Mit dem Smartphone dem Planeten helfen. Verfügbar unter https://www.otto.de/updated/ratgeber/umwelt-apps-mit-dem-smartphone-dem-planeten-helfen-36088, zuletzt geprüft am 07.05.2020.

Pohl, Lucas (2020): fridays for future. Verfügbar unter https://fridaysforfuture.de/, zuletzt geprüft am 13.04.2020.

Rogall, Holger (2012): Nachhaltige Ökonomie - Ökonomische Theorie und Praxis einer nachhaltigen Entwicklung. 2. Auflage. Marburg: Metropolis Verlag.

Sacher, Heiko. (2020): Studioresilience.com. Verfügbar unter htpps://www.Studioresilience. com, zuletzt geprüft am 07.05.2020.

Sauerhammer, Markus; Schwarz, Antonis; Zubrod, Andreas (2019): Nachrichtenlose Assets. Reformvorschlag. Verfügbar unter https://www.send-ev.de/uploads/sif.pdf, zuletzt geprüft am 03.05.2020.

Strigl, Alfred (2014): Soziale Innovationen - der Schlüssel zum Umwelt- und Klimaschutz? Verfügbar unter https://blog.ksoe.at/soziale-innovationen-der-schluessel-zum-umweltund-klimaschutz/, zuletzt geprüft am 02.05.2020.

Thiem, Carolin (2020): Es geht darum, dass man was tut. Zur Transformation von issues in Partizipationsprozessen. München: Universitätsbibliothek Technische Universität München.

Wehling, Peter (2012). From invited to uninvited participation (and back?): rethinking civil society engagement in technology assessment and development. Poiesis \& Praxis: International Journal of Ethics of Science and Technology Assessment, 9(1-2), 43-60. https://doi. org/10.1007/s10202-012-0125-2 


\section{(c) (1)}

Dieses Kapitel wird unter der Creative Commons Namensnennung 4.0 International Lizenz http://creativecommons.org/licenses/by/4.0/deed.de) veröffentlicht, welche die Nutzung, Vervielfältigung, Bearbeitung, Verbreitung und Wiedergabe in jeglichem Medium und Format erlaubt, sofern Sie den/die ursprünglichen Autor(en) und die Quelle ordnungsgemäß nennen, einen Link zur Creative Commons Lizenz beifügen und angeben, ob Änderungen vorgenommen wurden.

Die in diesem Kapitel enthaltenen Bilder und sonstiges Drittmaterial unterliegen ebenfalls der genannten Creative Commons Lizenz, sofern sich aus der Abbildungslegende nichts anderes ergibt. Sofern das betreffende Material nicht unter der genannten Creative Commons Lizenz steht und die betreffende Handlung nicht nach gesetzlichen Vorschriften erlaubt ist, ist für die oben aufgeführten Weiterverwendungen des Materials die Einwilligung des jeweiligen Rechteinhabers einzuholen. 